\title{
Etiopatogenia y diagnóstico de la boca seca
}

\section{Etiopathogenesis and diagnosis of dry mouth}

\author{
De Luca Monasterios FM**, Roselló Llabrés X*
}

\section{RESUMEN}

La saliva es esencial para mantener el equilibrio en la boca. Su ausencia o cambios en sus características representarían un problema importante de salud. La producción obedece a reflejos condicionados, la naturaleza del estímulo y una respuesta según la glándula. Los componentes de la saliva son de gran importancia ya que cumple numerosas funciones, entre ellas: formar y lubricar el bolo alimenticio para su deglución, mejorar el gusto, inicia la digestión, evita erosión de las mucosas, lubricar, mejora la capacidad de hablar, previene la deshidratación de las células epiteliales y receptores gustativos, mantiene equilibrio bacteriano, y un efecto de remineralización dental. La xerostomía es causada por disminución o ausencia de la secreción salival en condiciones de reposo, sin embargo por sí misma no es una enfermedad sino un síntoma; no parece estar relacionada directamente con la edad, sino con las enfermedades o fármacos que se suelen administrar en esa etapa de la vida. Algunos autores han demostrado que la xerostomía es un predictor importante en la pérdida involuntaria de peso en la tercera edad, y un déficit nutricional, que provoca deterioro de la función de las glándulas salivales, que repercute sobre los dientes con la consecuencia de edentulismo prematuro y deterioro de la salud, creándose así un círculo vicioso. De las distintas patologías orales que afectan a la población, la xerostomía se encuentra de segundo lugar después del síndrome de ardor bucal. Las causas son múltiples, englobadas en tres grandes grupos: orgánicas, farmacológicas y funcionales. Se dividen también en causas reversibles (ansiedad, infección aguda en las glándulas salivales, deshidratación o efecto secundario de algunos medicamentos) o irreversibles, si la causa es crónica, que conduce a una sequedad de boca permanente (anomalías congénitas, enfermedades autoinmunes, infecciosas, reumatológicas, menopausia, radioterapia, diabetes, trastornos psiquiátricos, Alzheimer, SIDA, consumo de sustancias adictivas). El diagnóstico de la hipofunción se basa en datos derivados de la sintomatología que refiere el paciente, destacando la necesidad de una amplia historia clínica, de la exploración mediante la constatación de los signos clínicos, de la medición del flujo salival o sialometría cuantitativa y de las pruebas complementarias según sea el caso. Por todo ello cabe destacar la importancia en la determinación de la afectación glandular (reversible o no) a tiempo temprano, y la instauración de medidas preventivas, de esta manera los pacientes tendrían un mejor pronostico y una mantenimiento de su calidad de vida.

Palabras clave: Saliva, funciones, boca seca, xerostomía, etiopatogenia, causas, diagnóstico, pruebas complementarias.

\section{SUMMARY}

Saliva is essential to maintain balance in the mouth. Its absence or changes in its characteristics represent an important health problem. Production is due to conditioned reflexes, the nature of the stimulus and response according to the gland. The components of saliva are of great importance since it has many functions, including: forming and lubricate the food bolus for deglutition, improve taste, starts digestion, prevents erosion of mucous, lubrication, improves the ability to speak, prevent dehydration of the epithelial cells and taste receptors, bacterial balance maintained, and an effect of dental remineralization. Xerostomia is caused by decreased or absent salivary secretion under resting conditions, however by itself is not a disease but a symptom, does not seem to be directly related to age, but to diseases or drugs that are usually given in that

* $\quad$ DDS, Máster en Medicina Bucal. Facultad de Odontología. Universidad de Barcelona.

** PhD, DDS, MD. Profesor Asociado Medicina Bucal. Facultad de Odontología. Universidad de Barcelona. 
stage of life. Some authors have shown that xerostomia is a major predictor of unintentional weight loss in the elderly, and nutritional deficits, which causes impairment of the function of the salivary glands, which affects the teeth with the consequence premature edentulism and deteriorating health, creating a vicious circle. Of the various oral diseases affecting the population of xerostomia is second only to burning mouth syndrome. The causes are multiple, included in three groups: organic, pharmacological and functional. Or irreversible; (anxiety, acute infection in the salivary glands, dehydration or side effect of some medications) are also divided into reversible causes, if the cause is chronic, leading to a permanent dry mouth (congenital anomalies, autoimmune diseases, infectious, rheumatologic, menopause, radiation, diabetes, psychiatric disorders, Alzheimer's, AIDS, substance abuse). Diagnosis of hypofunction is based on data derived from the symptoms reported by the patient, highlighting the need for a comprehensive medical history, exploration by a finding of clinical signs, measurement of salivary flow quantitative sialometry and additional tests as appropriate. Therefore worth highlighting the importance in the determination of glandular involvement (reversible or not), early in time, and the introduction of preventive measures, so patients would have a better prognosis and maintaining your quality of life.

Key words: Saliva, functions, dry mouth, xerostomia, causes, etiopathogenesis, diagnosis, additional tests.

Fecha de recepción: 15 de enero de 2014.

Aceptado para publicación: 19 de marzo de 2014.

De Luca Monasterios FM, Roselló Llabrés X. Etiopatogenia y diagnóstico de la boca seca. Av. Odontoestomatol 2014; 30 (3): 121-128.

\section{ETIOPATOGENIA}

\section{La saliva y sus funciones}

La saliva es esencial para mantener el equilibrio en la boca. Es un líquido incoloro, transparente, de escasa viscosidad, compuesto básicamente por agua entre $90 \%$ y 99,5\%, con un pH de 5,97 (en niños de $7,32)(1,2)$. Su ausencia o cambios en sus características representarían un problema importante de salud y se considera un mecanismo defensivo fundamental para proteger de agentes externos a la mucosa oral y a los dientes $(3,4)$.

Su producción obedece a reflejos condicionados por estímulos visuales, auditivos, olfativos, recuerdos, etc. La respuesta secretora es variable según la glándula y la naturaleza del estímulo (1). En condiciones normales, diariamente se segregan 1 a 1,5 litros; la cantidad de secreción se ve influida por otros motivos como son los ritmos circadianos y el ritmo de ingesta alimenticia, el tipo de alimentos ingeridos, el momento del ciclo menstrual, el embarazo y la menopausia, entre otras causas. Su viscosidad varía en función del equilibrio simpático-parasimpático. Las funciones y componentes de la saliva se resumen en la tabla 1. De manera genérica podemos decir que la saliva cumple con numerosas funciones, siendo un elemento clave para la homeostasis oral; contribuyendo a formar y a lubricar el bolo alimenticio para su deglución preparándolo para ser más digerible, además mejora la captación del gusto e inicia la digestión por acción directa de la amilasa salival $(1,5,6)$.

La lubricación del bolo evita que en su tránsito erosione las mucosas. Mejora la capacidad de hablar lubricando las mucosas, previene la deshidratación de las células epiteliales superficiales de las mucosas y de los receptores gustativos y mejora la reparación tisular. Mediante la autoclisis mantiene el equilibrio bacteriano, modificando la adherencia bacteriana (por acción de las inmunoglobulinas IgA, las mucinas, la lisocima y el calcio) y su papel tampón es imprescindible. Mantiene la integridad dentaria por su capacidad de remineralización $(1,5)$ (Tabla 1).

La secreción salival global es un parámetro variable, teniendo lugar en los 3 pares de glándulas salivales mayores y las glándulas salivales menores $(6,7)$. Por 
TABLA 1.- COMPONENTES DE LA SALIVA Y SUS FUNCIONES (5)

\begin{tabular}{|l|l|}
\hline Funciones & Componentes \\
\hline Lubricación & Mucina, glicoproteínas ricas en prolina, agua. \\
\hline Antimicrobiana & $\begin{array}{l}\text { Lisocima, lactoferrina, lactoperoxidasas, mucinas, cistinas, } \\
\text { histatinas, inmunoglobulinas, proteínas ricas en prolina, } \\
\text { IgA. }\end{array}$ \\
\hline Mantenimiento integridad de la mucosa & Mucinas, electrolitos, agua. \\
\hline Limpieza & Agua. \\
\hline Capacidad tampón y remineralización & $\begin{array}{l}\text { Bicarbonato, fosfato, calcio, staterina, proteínas aniónicas } \\
\text { ricas en prolina, flúor. }\end{array}$ \\
\hline Preparación de los alimentos para la deglución & Agua mucinas. \\
\hline Digestión & Amilasa, lipasa, ribonucleasa, proteasas, agua, mucinas. \\
\hline Sabor & Agua, gustina. \\
\hline Fonación & Agua, mucina. \\
\hline
\end{tabular}

término medio el volumen secretado es de $750 \mathrm{ml} /$ día, la mitad en reposo y la otra mitad durante la ingesta (1). En reposo el volumen de saliva se reparte entre las diferentes glándulas (Tabla 2). Las glándulas salivales pueden presentar diferentes patologías: anomalías del desarrollo, traumatismos, trastornos obstructivos, quistes, infecciones, enfermedades de origen metabólico, lesiones linfoepiteliales benignas y neoplasias. Algunos de estos procesos requerirán tratamiento médico y otros, quirúrgico $(1,8)$ (Tabla 2).

\section{Xerostomía}

Se define como sequedad de la boca causada por disminución o ausencia de la secreción salival en condiciones de reposo. La xerostomía por sí misma no es una enfermedad sino un síntoma que se presenta en diversas condiciones patológicas, ya sea como efecto secundario a la radiación de cabeza y cuello, a la ingesta de algunos medicamentos o a la disminución de la función de las glándulas salivales (6). Tal y como cita Bascones et al (3), la Federación Dental Internacional define a la Xerostomía como "la enfermedad del hombre moderno" debido a su carácter casi epidémico, siendo de esta manera un pro- blema con gran significación a la que se enfrentan pacientes y profesionales de la salud (9) teniendo múltiples consecuencias para la salud oral y la calidad de vida (6). Es una queja frecuente en la consulta, afectando principalmente pacientes ancianos $(2,6)$ llegando estos a alcanzar hasta un $20 \%$ (2). Sin embargo, por sí misma no parece estar relacionada directamente con la edad, sino con las enfermedades o fármacos que se suelen administrar en esa etapa de la vida $(3,6)$. Se habla de hiposecreción salival (sialopenia, hiposialia) cuando las cifras son menores a $0,1-0,2 \mathrm{ml} / \mathrm{min}$ (500 cc/día) en reposo y 0,5-0,7 $\mathrm{ml} / \mathrm{min}$ en saliva estimulada (3).

\section{TABLA 2.- VOLUMEN SALIVAL EN REPOSO SEGÚN LA GLÁNDULA SECRETORA $(1,9)$}

\begin{tabular}{|l|l|r|}
\hline Glándulas & Tipo de saliva & Volumen \\
\hline Submandibulares & Mucoserosa & $70 \%$ \\
Parótidas & Serosa & $20 \%$ \\
Sublinguales & Mucosa & $5 \%$ \\
Salivales menores & Mixta (predominio & $5 \%$ \\
& mucoso) & 5 \\
\hline
\end{tabular}


Clásicamente se acepta que la boca seca o Xerostomía afecta a 1 de cada 4 personas, aumentando, como ya hemos indicado, a partir de los cincuenta años. Hasta hace poco, se creía que la Xerostomía era patrimonio de la tercera edad, pero en estudios realizados en el norte de Europa, se encontró que entre el 20 y el $30 \%$ de las personas de veinte años, tienen esta patología. Aspecto que puede provocar edentulismo prematuro; pudiendo estar relacionado con el creciente consumo de antidepresivos, bebidas alcohólicas y tabaco (3).

La Xerostomía es más frecuente en el sexo femenino (10), principalmente por los cambios hormonales asociados al embarazo, climaterio o a la menopausia. Hasta un $80 \%$ de las mujeres menopáusicas padecen sequedad en las mucosas (3). Diferentes estudios han evaluado las distintas patologías orales que afectan a la población, estando la xerostomía en el segundo lugar después del síndrome de ardor bucal $(23,4 \%)$ entre los diagnósticos más frecuentes con un $10,6 \%$ de los casos (10).

Según las estadísticas, sólo uno de cada 1.500 pacientes acude al médico u odontólogo por motivo de la sequedad, pero cuando se interroga sobre el problema uno de cada 10 pacientes afirma presentar sequedad de boca (2). Esto es importante si pensamos en las consecuencias a medio y largo plazo que tiene la persistencia de la boca.

\section{Causas de xerostomía}

La disminución del flujo salival se debe a una hipofunción de las glándulas salivales (8). Esta disminución de la cantidad de saliva puede ser reversible si la causa que la desencadena es pasajera, como un estado de ansiedad, una infección de tipo aguda en las glándulas salivales, un cuadro de deshidratación o como efecto secundario de algunos medicamentos $(1,6,11)$. En cambio, si la causa es de tipo cróni$\mathrm{co}$, conduce a una sequedad de boca permanente, como ocurre en algunas anomalías congénitas, en enfermedades autoinmunes, infecciosas, reumatológicas (síndrome de Sjögren primario o secundario), enfermedades alérgicas, menopausia, radioterapia en cáncer primordialmente de cabeza y cuello, diabetes, trastornos psiquiátricos, Alzheimer, SIDA, consumo de sustancias adictivas (alcohol, drogas y tabaco) $(3,12,7)$.

Las causas se agrupan en cuatro categorías: Orgánicas, farmacológicas, funcionales y otras. Las repasaremos brevemente (7).

- Causas orgánicas: Hay varias enfermedades sistémicas que pueden cursar con xerostomía, las más frecuentes las presentamos en la Tabla 3.

- Causas farmacológicas: Los efectos secundarios de los fármacos son los que provocan la mayoría de los casos de xerostomía $(7,11)$ (Figura 1). La actividad de las glándulas salivales está regulada por el sistema nervioso vegetativo y dispone de receptores colinérgicos muscarínicos, así pues todos los fármacos con acción anticolinérgica o que produzcan bloqueo de los receptores producen una disminución de la salivación (7). Existen más de cuatrocientos medicamentos que inducen a una hipofunción de las glándulas salivales, como efecto adverso potencial, siendo éste uno de los principales motivos de abandonos de la medicación por parte de los pacientes. Hay que reseñar, que estos fármacos además de presentar un elevado índice de consumo, son en su mayoría la terapia de elección en los tratamientos de enfermedades de tipo crónico o de carácter epidémico. Sobre todo de los que tienen efectos antimuscarínicos con acción atropínica, como los antidepresivos tricíclicos, antihistamínicos o

\section{TABLA 3.- LISTADO DE ENFERMEDADES SISTÉMICAS QUE PUEDEN CAUSAR XEROSTOMÍA $(3,7,8)$}

Enfermedades sistémicas y xerostomía

- Síndrome de Sjögren

- Amiloidosis

- Sarcoidosis

- Infección por VHC y VIH

- Parotiditis transitoria (viral o bacteriana)

- Cirrosis biliar

- Fibrosis quística

- Diabetes mellitus

- Agenesia glandular

- Depósito de hierro (talasemia) 
por sobredosis de diuréticos, cuyo uso actualmente va en aumento (Tabla 4) $(3,7,11)$.

- Causas funcionales: Otros factores que producen cambios en el fluido o en el balance electrolítico de la saliva son la deshidratación, la privación o pérdida de líquidos, la diarrea y/o vómitos persistentes, los déficits proteínicos, las alteraciones cardíacas, la uremia y el edema. Además, el transporte de saliva se puede afectar por obstrucciones (sialolitiasis), infecciones (sialoadenitis) y estenosis en los conductos y conductillos. La xerostomía puede deberse a un mayor consumo de saliva por causas inespecíficas, como respirar por la boca debido a obstrucción nasal, utilizar inhaladores y padecer estrés, estados de ansiedad o depresión severa (7).

- Otras causas: Son de destacar el alcohol y el tabaco.

\section{DIAGNÓSTICO DE LA XEROSTOMÍA}

El diagnóstico de la hipofunción se basa en datos derivados de la sintomatología que refiere el paciente, de la exploración clínica, mediante la constatación de los signos clínicos, de la medición del flujo salival o sialometría cuantitativa y de las pruebas complementarias según sea el caso (Tabla 5).

\section{TABLA 4.- GRUPOS DE MEDICAMENTOS XEROGÉNICOS (7)}

\footnotetext{
Fármacos xerostomizantes

- Antidepresivos tricíclicos

- Antiespasmódicos

- Neurolépticos

- Inhibidores de la MAO

- Agentes antiparkinsonianos

- Litio

- Agonistas adrenérgicos centrales

- Diuréticos

- Descongestionantes nasales

- Antihistamínicos

- Broncodilatadores

- Descongestivos - anticatarrales

- Anorexígenos (anfetaminas)
}

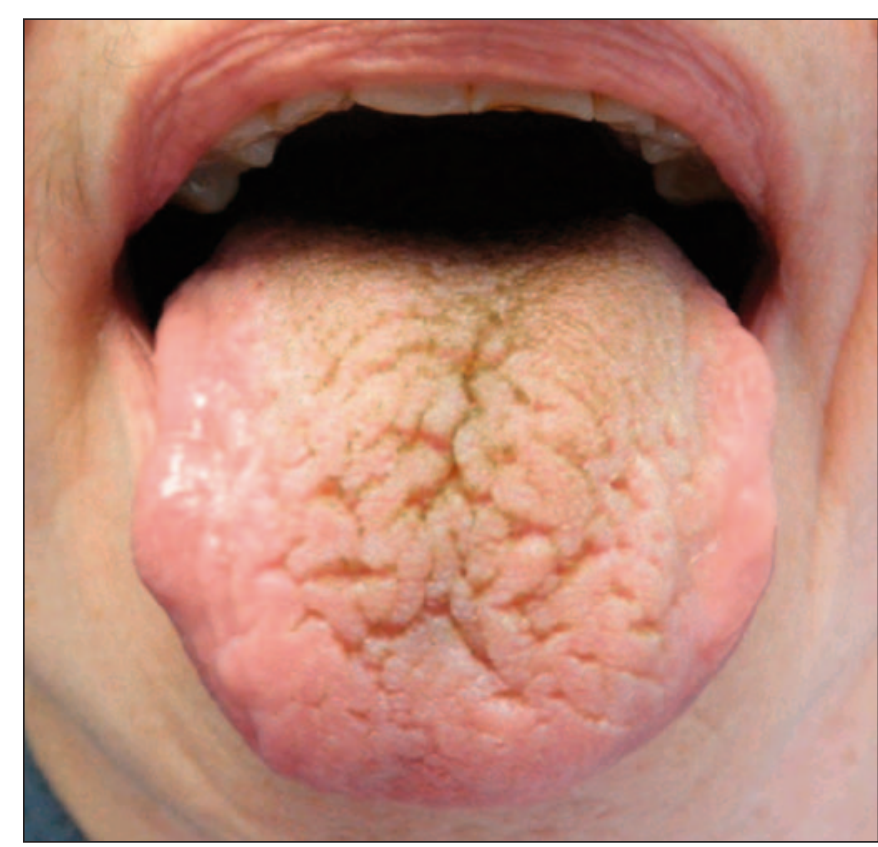

Fig. 1. Lengua fisurada, depapilada y saburral, con síntomas de ardor, disgeusia y halitosis en paciente con xerostomía asociada a medicamentos antidepresivos.

\section{TABLA 5.- PRINCIPALES SIGNOS Y SÍNTOMAS DE SEQUEDAD BUCAL}

\section{Sintomatología}

- Saliva viscosa, pegajosa, espumosa.

- Dificultad de hablar y tragar

- Sequedad de labios, lengua y mucosas

- Halitosis (mal aliento)

- Lesiones traumáticas orales

- Fisuras

- Lengua depapilada

- Queilitis angular

- Mucosa eritematosa

- Candidiasis oral recidivante

- Aumento de caries dental

- Erosiones dentarias, mayor sensibilidad

- Acumulación de placa

- Gingivitis

- Alteración de la dieta

- Sensación de quemazón oral

- Reflujo ácido acentuado 
Para el diagnóstico se recomiendan la combinación de técnicas clásicas y modernas (13), destacando la necesidad de una amplia historia clínica. Tanto los profesionales como investigadores han recurrido a escalas visuales analógicas, encuestas de calidad de vida y a cuestionarios $(4,12)$, similares al utilizado en la evaluación de condiciones subjetivas, como es el dolor (3). A través de estos cuestionarios el paciente hace una autovaloración sobre la enfermedad, y después podremos ahondar en la anamnesis y en diferentes pruebas complementarias para llegar a un diagnóstico en función del cual podemos instaurar un tratamiento individualizado. Los tratamientos básicamente se centran en la prevención, la etiología, los signos y síntomas, estimulación salival de manera mecánica-local y los tratamientos sistémi$\cos (15)$.

En base a esto, incluimos un cuestionario específico para encaminar el interrogatorio de pacientes con sequedad bucal (Tabla 6). Muchos pacientes con xerostomía no refieren de entrada sequedad bucal, aunque reconocen que tienen algún tipo de molestia como disfagia a alimentos secos (7).

\section{TABLA 6.- CUESTIONARIO PARA PACIENTES CON XEROSTOMÍA}

- ¿Qué medicamentos está tomando?

- ¿Consume suplementos alimenticios?

- ¿Usa prótesis dental (de qué tipo) y cada cuándo la limpia?

- ¿Con qué frecuencia se lava los dientes?

- ¿Le duele la boca o los dientes?

- ¿Para humedecer la boca bebe sorbos de agua frecuentemente?

- ¿El chupar caramelos le mejora la sensación?

- ¿Siente la boca seca en el inicio de las comidas?

- ¿Percibe que tiene menos saliva que antes?

- ¿Le cuesta trabajo deglutir (tragar)?

- ¿Le es difícil consumir alimentos secos como galletas, pan o pan tostado?

- ¿Se levanta en mitad de la noche para beber líquidos?

- ¿Tiene seca la garganta, ojos o el interior de la nariz?
- Inspección: Observar si existen asimetrías, piel normal o enrojecida. Es importante la inspección intrabucal de la salida de los conductos Stenon, Wharton o Bartolini para evaluar si están tumefactos, dilatados o se evidencian cálculos (1).

- Palpación: Será importante valorar si existe tumefacción, contrastando la consistencia de la glándula contralateral, pudiendo ser uni o bilateralidad, extra e intrabucal, cambio de temperatura, dolor, superficie lisa o irregular, firme o blanda, móvil o adherida a planos profundos con contenido líquido o no (1).

- Analítica general: Nos permite valorar el estado general, nutricional y el estado de la función metabólica del paciente.

- Estudio de la secreción salival: Suele ser útil en pacientes que refieren sequedad bucal. Existen varias técnicas para medir el flujo salival $(7,15)$. La sialometría en reposo resulta muy útil. Una manera sencilla es el pesaje de un algodón antes y después de haberlo colocado debajo de la lengua durante 5 minutos o bien depositando el contenido salival en un recipiente milimetrado. Existen pruebas más elaboradas que nos permiten analizar la secreción. De entre ellas destaca el test Oral de Schirmer's (8), se realiza colocando una tira de papel de filtro milimetrada en el suelo de la boca, cercana a la salida del conducto de Wharton, durante 5 min., la medición se realiza a partir de la longitud humedecida. El flujo medio de saliva $40,92 \pm 22: 28 \mathrm{~mm} / 5 \mathrm{~min}$. en pacientes sanos.

Para medir el flujo salival estimulado, se puede hacer mediante estimulación mecánica (mediante la masticación de parafina, chicles, etc.) o química con ácido cítrico $4 \%$. Se considera que el flujo de saliva no estimulada inferior a $0,15 \mathrm{~mL} /$ minuto o estimulada de $0,2 \mathrm{a} 0,18 \mathrm{~mL} /$ minuto es anormal, pero no causante de xerostomía $(8,15)$.

- La sialografía consiste en la inyección de medio de contraste radiopaco (1-2 ml) de manera ambulatoria introducido a través del conducto principal secretor (Stennon o Wharton). El contraste se distribuye por todo el sistema de conductos y a continuación se realizan radiografías de frente y de perfil para conocer si los conductos y conductillos salivales están permeables o existe obstrucción por sialolitos o tumores sólidos. Desde la introducción de técnicas más nuevas la sia- 
lografía ha perdido valor parte en el estudio diagnóstico de la patología salival (1).

- La escintigrafía o gammagrafía es una técnica diagnóstica auxiliar no invasiva. Permite valorar las zonas no funcionantes. Se utiliza como trazador el tecnecio-99, que permite visualizar las glándulas salivales (su tamaño, la posición y la forma) además de informar sobre su mecanismo excretor debida a la captación y excreción del radioisótopo (1).

- La biopsia incisional de las glándulas salivales accesorias o menores es un procedimiento para confirmar el diagnóstico. Es prueba muy fiable. Se trata de un método relativamente sencillo, tras la anestesia infiltrativa local del labio inferior se realiza una incisión pequeña y horizontal sobre la mucosa labial y se diseccionan las glándulas salivales menores. El foco que se debe tener en cuenta para el estudio anatomopatológico es de $5 \mathrm{~mm}^{2}$, que equivale a un área de cinco glándulas salivales menores. Es una técnica reconocida para el diagnóstico del Síndrome de Sjogren (7). En el examen histopatológico, se encontrará infiltración linfoidea, atrofia acinar e hipertrofia del epitelio ductal, con obstrucción gradual de la luz y desarrollo de islotes de células mioepiteliales formadas a partir del epitelio ductal proliferante. Se pueden definir diversos grados inflamatorios dependiendo de la gravedad. Se considera positiva cuando se observa 1 foco/5 $\mathrm{mm}^{2}$ de tejido glandular constituido por 50 o más linfocitos $(1,7)$. Otras técnicas auxiliares son la biopsia por punción con aguja fina (PAAF) con jeringa de 20 $m L$. Es de elección en el estudio de masas salivales y tiene una elevada fiabilidad en el diagnóstico de los tumores de las glándulas salivales (95\%) aunque de menor eficacia en las enfermedades no tumorales $(1,8)$. Es importante informar al patólogo de la clínica y las pruebas complementarias previamente realizadas.

\section{Otras pruebas de imagen}

La radiografía convencional o simple es útil en el estudio de una masa glandular por ejemplo si se trata de cálculos. Una prueba de rayos $\mathrm{X}$ común puede detectar y mostrar la posición de 8 de cada 10 cálculos salivales. Sin embargo en 2 de cada 10 casos, el cálculo no es tan evidente en una placa de rayos $\mathrm{X}$ y se puede necesitar otras pruebas. Así la Ortopantomografía, la Radiografía oclusal y la Radiografía periapical son útiles para el estudio de la litiasis del conducto de Wharton, objetivándose su forma, tamaño y posición (1).

La Tomografía computarizada y la Resonancia magnética resultan útiles en neoplasias malignas o recurrentes, neoplasias grandes o afectación de otros espacios (1). La TC se efectúa ante la sospecha de un tumor de las glándulas salivales ofreciendo con frecuencia criterios diagnósticos de benignidad o malignidad (1). La Resonancia magnética tiene las mismas indicaciones del TC, solo que tiene la ventaja que no produce radiación (1). La Ecografía de alta resolución aporta datos sobre las características de la masa: sólida, quística, uni o multifocal y su delimitación. La información que aporta en comparación con los otros métodos es escasa. Posee ciertas ventajas como la ausencia de radiación, bajo coste y rapidez en el examen.

A modo de conclusiones la sensación de boca seca es una queja muy común en pacientes, pero no por ello menos importante. Algunos autores han demostrado que la xerostomía es un predictor importante para la pérdida involuntaria de peso en la tercera edad y este déficit nutricional provoca deterioro de la función de las glándulas salivales, que repercute sobre los dientes. Por consiguiente se agrava el déficit nutritivo y el estado general del individuo, creándose un círculo vicioso.

El Diagnóstico definitivo exige la conjunción de datos aportados por la clínica (anamnesis y exploración), unido a pruebas complementarias (imagen, PAAF y Biopsia).

Finalmente la xerostomía no debe ser considerada como intrascendente sino abordarse con los recursos terapéuticos al alcance del médico u odontólogo. Para prevenir y tratar la xerostomía es fundamental mantener una adecuada salud bucodental.

\section{BIBLIOGRAFÍA}

1. Chimenos Küsner, Eduardo. Historia Clínica en Odontología. Barcelona: Masson 1998. 
2. Gallardo JM. Xerostomía: etiología, diagnóstico y tratamiento. Rev Med Inst Mex Seguro Soc 2008;46:109-16.

3. Bascones A, Tenovuo J, Ship J, Turner M, MacVeigh I, López-Ibor JM, et al. liaga A. Conclusiones del Simposium 2007 de la Sociedad Española de Medicina Oral sobre "Xerostomía. Síndrome de Boca Seca. Boca Ardiente". Av. Odontoestomatol 2007;23:119-26.

4. Pai S, Ghezzi EM, Ship JA. Development of a Visual Analogue Scale questionnaire for subjective assessment of salivary dysfunction. Oral Surg Oral Med Oral Pathol Oral Radiol Endod 2001;91:311-6.

5. Sreebny L, Baum B, Edgar W, Epstein J, Fox P, Llamas M. Saliva: Its role in health and diseases. Int Dent J 1992;42:291-304.

6. Gupta A; Epstein JB, Sroussi H. Hyposalivation in elderly patients. J Can Dent Assoc 2006;72: 841-6.

7. Cawson RA, Odell EW. Fundamentos de medicina y patología oral. $8^{a}$ edición. Barcelona: Editorial Elsevier España 2009.

8. López-Jornet P. Alteraciones de las glándulas salivales. Universidad de Murcia. España. Servicio de publicaciones 2002. Pag 17-38.

9. Dost F, Farah CS. Stimulating the discussion on saliva substitutes: a clinical perspective. Aust Dent J 2013;58:11-17.

10. Brailo V, Boras VV, Pintar E, Juras DV, Karaman $\mathrm{N}$, Rogulj AA. [Analysis of oral mucosal lesions in patients referred to oral medicine specialists]. Lijec Vjesn 2013;135:205-8.

11. Gueiros LA, Soares MS, Leão JC. Impact of ageing and drug consumption on oral health. Gerodontology 2009;26: 297-301.

12. Henson BS, Inglehart MR, Eisbruch A, Ship JA. Preserved salivary output and xerostomia-related quality of life in head and neck cancer patients receiving parotidsparing radiotherapy. Oral Oncol 2001;37:84-93.

13. Jacobson JJ. Is dentistry going to get into the salivary diagnostics game or watch from the sidelines? Todays FDA 2013;25:26-7:29-33.

14. López-López J, Jané Salas E. Chimenos Küstner E. Prognosis and treatment of dry mouth. Systematic review. Med Clin (Barc) 2014;142: 119-24.

15. López Jornet P, Camacho-Alonso F, BermejoFenoll A. A simple test for salivary gland hypofunction using Oral Schirmer's test. J Oral Pathol Med 2006;35:244-8.

\section{CORRESPONDENCIA}

Dr. Xavier Roselló Llabrés

Campus Universitario de Bellvitge

Departamento de Odontoestomatología

Facultad de Odontología

Pabellón de Gobierno

Feixa Llarga, s/n

08907 L'Hospitalet de Llobregat. Barcelona

Correo electrónico: xavier_rosello@hotmail.com 